\title{
Enfermedad de Crohn fibroestenótica en íleon medio como manifestación inicial de la enfermedad. Reporte de un caso
}

\section{A Case Report of Fibro-Stenotic Crohn's Disease in the Middle Ileum as the Initial Manifestation}

Adriana Margarita Rey, ${ }^{1}$ Gustavo Reyes, ${ }^{1}$ Fernando Sierra, ${ }^{1}$ Rafael García-Duperly, ${ }^{2}$ Rocío López, ${ }^{3}$ Leidy Paola Prada. ${ }^{4}$

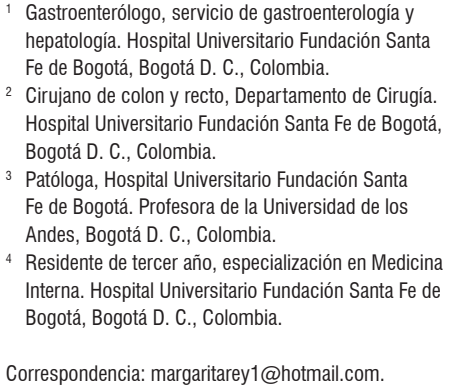
hepatología. Hospital Universitario Fundación Santa Fe de Bogotá, Bogotá D. C., Colombia.

2 Cirujano de colon y recto, Departamento de Cirugía. Hospital Universitario Fundación Santa Fe de Bogotá, Bogotá D. C., Colombia.

3 Patóloga, Hospital Universitario Fundación Santa Fe de Bogotá. Profesora de la Universidad de los Andes, Bogotá D. C., Colombia.

${ }^{4}$ Residente de tercer año, especialización en Medicina Interna. Hospital Universitario Fundación Santa Fe de Bogotá, Bogotá D. C., Colombia.

Correspondencia: margaritarey1@hotmail.com.

Fecha recibido: $\quad 06-04-17$ Fecha aceptado: 06-10-17

\begin{abstract}
Resumen
La enfermedad de Crohn (EC) es una enfermedad inflamatoria intestinal que puede afectar todo el tracto gastrointestinal; el intestino delgado es el segmento afectado en cerca del $50 \%$ de los pacientes, en los cuales el íleon terminal es el área más comúnmente comprometida por la enfermedad.

Las estenosis intestinales son una complicación frecuente en la EC y aproximadamente $30 \%$ a $50 \%$ de los pacientes presentan estenosis o lesiones penetrantes al momento del diagnóstico. Las técnicas endoscópicas convencionales no permiten evaluar las lesiones del intestino delgado; es por esto que se desarrollaron técnicas como la enteroscopia y la videocápsula endoscópica, teniendo cada una de ellas sus ventajas e indicaciones.

Se presenta un caso de un paciente con EC con fibroestenosis localizada en el íleon medio. No es una localización frecuente para este tipo de lesiones.
\end{abstract}

\section{Palabras clave}

Enfermedad de Crohn, fibroestenosis, intestino delgado, videocápsula endoscópica, enteroscopia.

\begin{abstract}
Crohn's disease $(C D)$ is an inflammatory bowel disease that can affect the entire gastrointestinal tract. The small intestine is affected in about $50 \%$ of patients among whom the terminal ileum is the area most commonly affected.

Intestinal stenosis is a common complication in CD and approximately $30 \%$ to $50 \%$ of patients present stenosis or penetrating lesions at the time of diagnosis. Because conventional endoscopic techniques do not allow evaluation of small bowel lesions, techniques such as enteroscopy and endoscopic video-capsule were developed. Each has advantages and indications.

We present the case of a patient with CD with localized fibrostenosis in the middle ileum which is not a frequent site for this type of lesion.
\end{abstract}

\section{Keywords}

Crohn's disease, fibrostenosis, small intestine, endoscopic video-capsule, enteroscopy.
La EC es una enfermedad inflamatoria intestinal crónica que causa síntomas como dolor abdominal, diarrea y deposiciones con sangre. Puede afectar cualquier parte del tracto gastrointestinal, comprometiendo en $40 \%$ a $60 \%$ de los casos el intestino delgado (1). En el intestino delgado, el íleon terminal es el área más comúnmente afectada. Es poco frecuente la localización proximal, la cual ha sido reportada hasta en un $10 \%$ de los pacientes (2).

Se presenta el caso de un hombre de 65 años sin antecedentes previos, que consultó por un cuadro de 24 horas de evolución de dolor y distensión abdominal generalizada asociada con vómito en "cuncho de café" documentándose 
obstrucción intestinal en radiografía simple de abdomen, que se confirmó en la tomografía con cambios estructurales inespecíficos en asas ileales distales, sin signos de sufrimiento de asa (Figura 1). En los paraclínicos complementarios se identificó la presencia de anemia microcítica hipocrómica, por lo que se volvió a interrogar al paciente y refirió episodios previos aislados de melenas con estudios de endoscopia de vías digestivas altas y colonoscopia total sin evidencia de lesiones patológicas.

Se realizó una videocápsula endoscópica para el estudio de sospecha de sangrado del intestino delgado, donde se documentó la presencia de estenosis y úlcera localizada en el íleon medio sin sangrado activo (Figura 2). Por estos hallazgos se programó para la realización de una enteroscopia de doble balón por vía retrógrada, donde se evidenció a $190 \mathrm{~cm}$, en forma ascendente a la válvula ileocecal, la presencia de estenosis concéntrica con luz aproximada de $8 \mathrm{~mm}$ de diámetro con ulceración en su borde libre sin sangrado, cubierta de fibrina, no franqueable (Figura 3). Durante la enteroscopia, se tomaron biopsias de la estenosis y se realizó un habón submucoso con abundante solución salina, posteriormente se marcó este punto a $3 \mathrm{~cm}$ distal al sitio de la estenosis con tinta china para facilitar su localización.
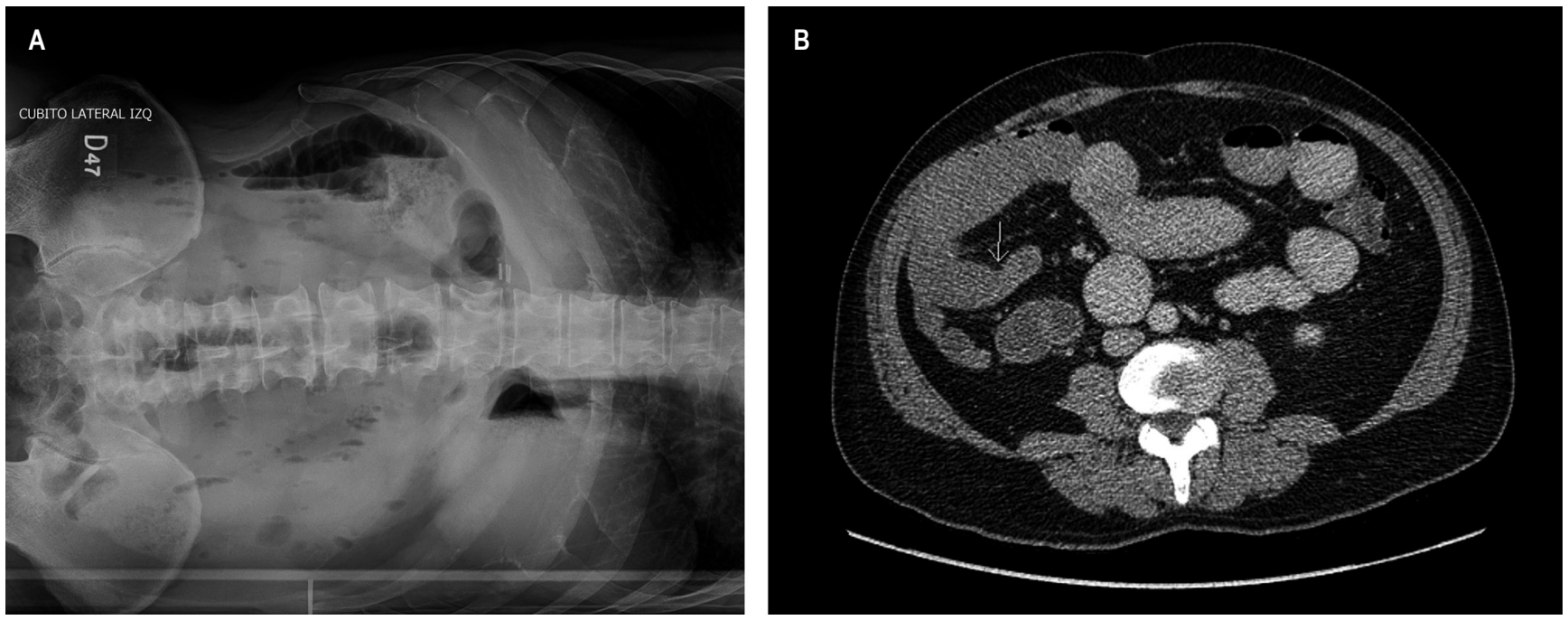

Figura 1. A. Radiografía de abdomen simple; y B. Corte sagital de tomografía de abdomen con contraste. Ambas imágenes muestran obstrucción intestinal y cambios estructurales no específicos en las asas ileales distales, sin signos de sufrimiento de asa.

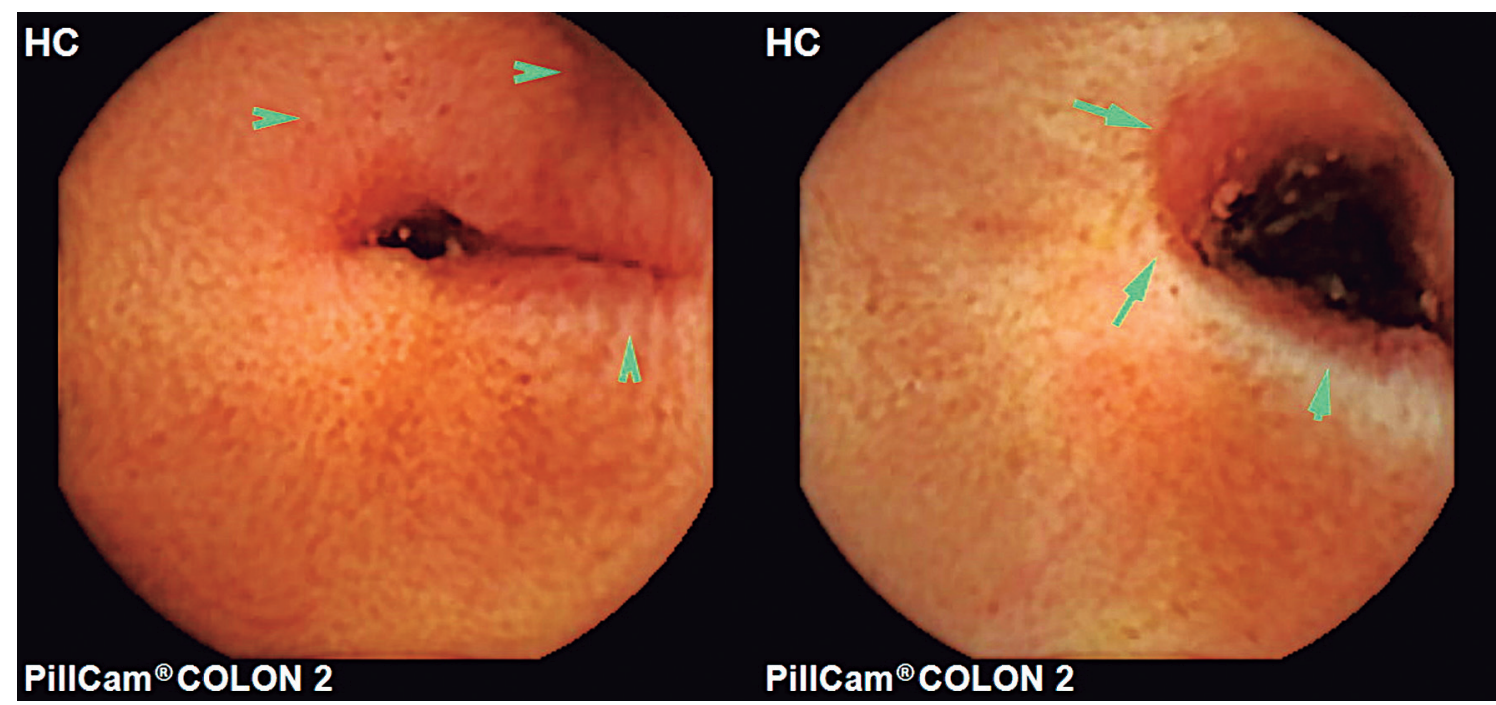

Figura 2. Videocápsula en la que se evidencia la presencia de estenosis y úlcera localizada en íleon medio sin sangrado activo. 
El reporte de patología informó que la lesión es compatible con EC. Se comentó el caso en la junta médica con la participación del servicio de gastroenterología y de cirugía de colon y recto; se decidió realizar una resección electiva quirúrgica de segmento estenótico, por haberse documentado fibroestenosis con alto riesgo de obstrucción sin evidencia de otras lesiones en el resto del intestino delgado.

Se realizó una cirugía por vía laparoscópica evidenciándose macroscópicamente la presencia de estenosis de íleon de aproximadamente $1 \mathrm{~cm}$ con infiltración grasa sobre la serosa y el tatuaje adyacente. El reporte de patología del espécimen quirúrgico describió la presencia de alteración de la arquitectura de la pared de intestino delgado, con pérdida focal de las vellosidades con ulceración, acortamiento y ensanchamiento velloso; criptas ligeramente distorsionadas con disminución de las células caliciformes, fibrosis de la lámina propia con aumento del infiltrado linfoplasmocitario, además de metaplasia de glándulas submucosas e hipertrofia de fibras nerviosas. Estos hallazgos son compatibles con enfermedad inflamatoria intestinal crónica de tipo EC en fase activa (Figura 4).

Durante el seguimiento ambulatorio, el paciente no ha presentado nuevos episodios de dolor abdominal ni síntomas sugestivos de obstrucción intestinal. Continúa en manejo con hierro oral por anemia ferropénica y los estudios endoscópicos realizados 1 año después del procedimiento se encontraron dentro de límites normales.

La incidencia de la EC ha aumentado en los últimos 50 años y su comportamiento clínico sigue siendo muy heterogéneo. Su diagnóstico debe realizarse teniendo en cuenta

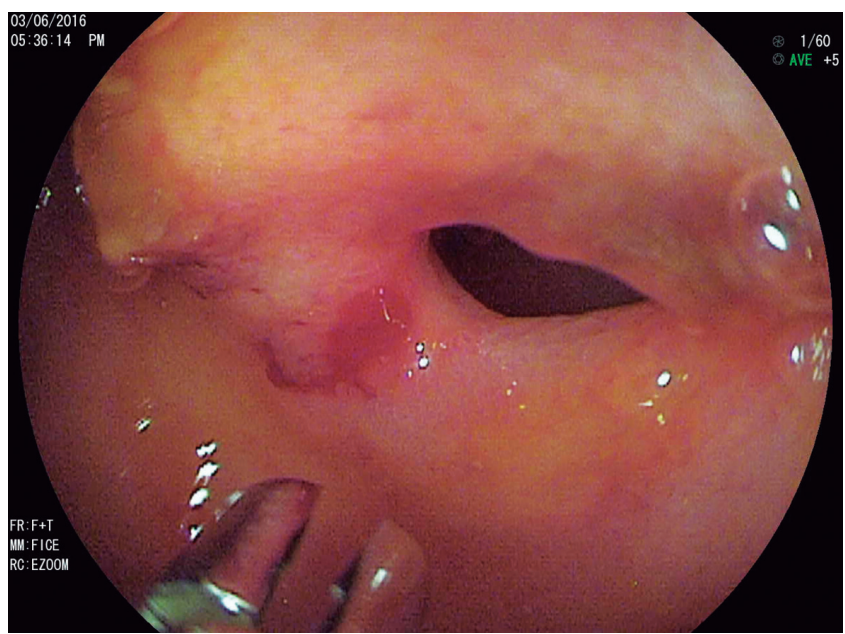

Figura 3. Enteroscopia por vía retrógrada en la que se observa una estenosis concéntrica de la luz a $190 \mathrm{~cm}$ en sentido ascendente de la válvula ileocecal con ulceración del borde libre sin evidencia de sangrado activo. los hallazgos clínicos, endoscópicos, histopatológicos e imagenológicos; es por esto que es indispensable la realización de estudios endoscópicos para la toma de muestras que permita confirmar el diagnóstico histológico de esta enfermedad $(1,3)$.

Las estenosis intestinales son una complicación frecuente en la EC, se ha descrito su presencia hasta en un $40 \%$ de los pacientes con enfermedad localizada en íleon (4) y aproximadamente $30 \%$ a $50 \%$ de los pacientes presentan estenosis o lesiones penetrantes al momento del diagnóstico (5). La relevancia clínica de estas estenosis se debe a que hasta en un $60 \%$ de los casos se requiere de cirugía dentro de los primeros 20 años posteriores al diagnóstico (6).

Dado que la EC es una enfermedad transmural, esta afecta todas las capas del intestino favoreciendo los depósitos de proteínas en la matriz extracelular que alteran la arquitectura de los segmentos comprometidos (5). Las estenosis son el resultado de la inflamación crónica que lleva a una remodelación tisular, hipertrofia de las células mesenquimales, hiperplasia y fibrosis de los segmentos afectados por la enfermedad inflamatoria intestinal; como consecuencia de un proceso alterado de cicatrización posterior a la lesión inicial y a la respuesta inflamatoria que se presenta en el paciente susceptible genéticamente $(1,7)$. Las estenosis con componente puramente inflamatorio se benefician del tratamiento con antiinflamatorios que disminuyen el edema. Como tratamiento complementario, está indicada la dilatación endoscópica y la cirugía en caso de que en las lesiones predomine el componente fibrótico, el cual no responde al tratamiento médico (3).

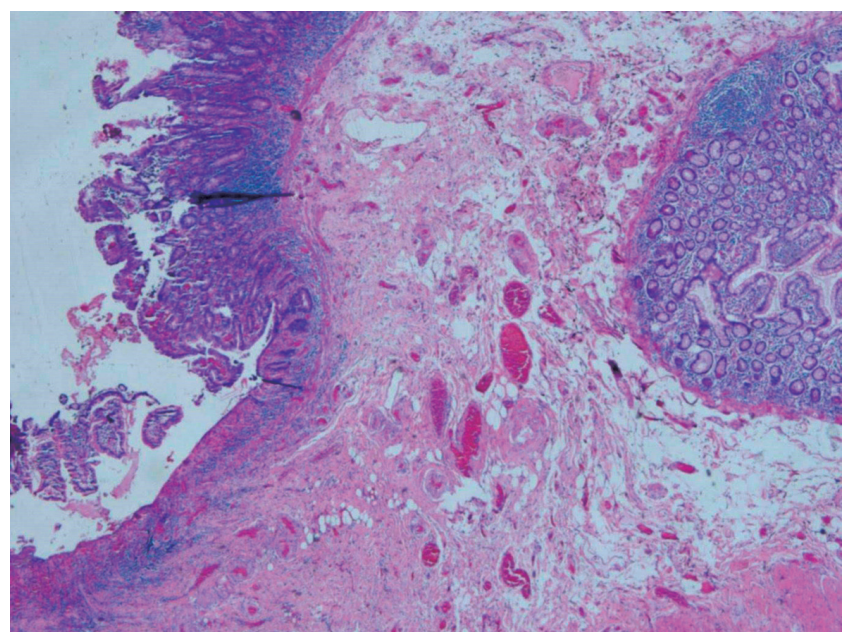

Figura 4. Hematoxilina y eosina 4X. Muestra de la pared del intestino con alteración de la arquitectura. A la izquierda de la microfotografía hay ulceración, con infiltrado linfoplasmocitario y formación de acúmulos linfoides, con área de fibrosis submucosa. A la derecha hay glándulas irregulares, con metaplasia pseudopilórica. 
Las técnicas endoscópicas convencionales no permiten evaluar las lesiones del intestino delgado secundarias a EC que se localizan distales al duodeno o proximales al íleon terminal; es por esto que se desarrollaron técnicas como la enteroscopia y la videocápsula endoscópica. La principal ventaja de la cápsula es que realiza una evaluación completa del intestino delgado con un alto rendimiento diagnóstico, pero no tiene la posibilidad de tomar muestras y se debe individualizar su indicación en casos que se sospechen estenosis por el riesgo de impactación. Las ventajas de la enteroscopia son que permite la toma de biopsias y la realización de procedimientos terapéuticos (8).

Teniendo en cuenta que los estudios endoscópicos solo permiten evaluar cuando hay compromiso endoluminal por la enfermedad, los estudios de imágenes pueden utilizarse como técnica complementaria ya que permiten evaluar el compromiso de la pared del intestino y de los tejidos adyacentes. El ultrasonido intestinal se enfoca en detectar el engrosamiento de la pared intestinal y el aumento de la vascularización por medio del Doppler, al igual que la presencia de estenosis y de lesiones extramurales. La enterografía por tomografía permite la detección de estenosis con o sin dilatación proximal, al igual que signos de enfermedad penetrantes como la presencia de fístulas o abscesos extraluminales; y la enterorresonancia tiene la ventaja de ser un método libre de radiación con diferentes protocolos de adquisición, que permite la evaluación cuantitativa y cualitativa de la actividad y severidad de la enfermedad. El método a escoger depende de la disponibilidad y experiencia en la realización de estos estudios (4).

Con respecto al tratamiento, en los casos de estenosis cortas, no complicadas, o de anastomosis ileocolónicas o colónicas se puede realizar una dilatación con balón por medio de endoscopia si se seleccionan adecuadamente los pacientes; sin embargo, se debe tener en cuenta el riesgo de perforación durante el procedimiento (5). Deben evitarse resecciones intestinales extensas debido a las posibles complicaciones relacionadas con el intestino corto, diarrea y malabsorción; es por esto que se han desarrollado técnicas más conservadoras limitadas a la resección de la estenosis seguida por un cierre longitudinal, siendo preferible el abordaje laparoscópico para disminuir la frecuencia de adherencias y hernias incisionales con mejor recuperación clínica $(9,10)$.

\section{REFERENCIAS}

1. Kim M, Jang HJ. The role of small bowel endoscopy in small bowel Crohn's disease: when and how? Intest Res. 2016;14(3):211-7. https://doi.org/10.5217/ir.2016.14.3.211

2. Van Assche G, Dignass A, Panes J, et al. The second European evidence-based Consensus on the diagnosis and management of Crohn's disease: Definitions and diagnosis. J Crohns Colitis. 2010;4(1):7-27.

3. Siegmund B. Medical Therapy of fibrostenotic Crohn's disease. Viszeralmedizin. 2015;31(4):259-64.

4. Bettenworth D, Nowacki TM, Cordes F, et al. Assessment of stricturing Crohn's disease: Current clinical practice and future avenues. World J Gastroenterol. 2016;22(3):1008-16.

5. Rieder F, Latella G, Magro F, et al. European Crohn's and colitis organisation topical review on prediction, diagnosis and management of fibrostenosing Crohn's disease. J Crohns Colitis. 2016;10(8):873-85.

6. Peyrin-Biroulet L, Harmsen WS, Tremaine WJ, et al. Surgery in a population-based cohort of Crohn's disease from Olmsted County, Minnesota (1970-2004). Am J Gastroenterol. 2012;107(11):1693-701.

7. Li C, Kuemmerle JF. Mechanisms that mediate the development of fibrosis in patients with Crohn's disease. Inflamm Bowel Dis. 2014;20(7):1250-8.

8. Park SJ, Kim WH. A look into the small bowel in Crohn's disease. Clin Endosc. 2012;45(3):263-8.

9. Thienpont C, Van Assche G. Endoscopic and medical management of fibrostenotic Crohn's disease. Dig Dis. 2014;32 Suppl 1:35-8. https://doi.org/10.1159/000367824

10. Nasr JY, Lloyd J, Yadav D. An unusual cause of fibrostenotic terminal ileal disease. Gastroenterology. 2011;141(3):e5-6. 